\title{
Linkage between Proteolysis and Conformational Change in Virus Assembly: Insights from Cryo-Electron Microscopy
}

\author{
J. F. Conway, * N. Cheng,† P. D. Ross,\# L. Dierkas,§ B. A. Firek,§ R. W. Hendrix,§ R. L. Duda, \\ and $\underline{\text { A. C. Steven }} \dagger$ \\ * Department of Structural Biology, U. Pittsburgh School of Medicine, Pittsburgh, PA 15260; \\ $\dagger$ Laboratory of Structural Biology, NIAMS, and \#Laboratory of Molecular Biology, NIDDK, NIH, \\ Bethesda, MD 20892; §Department of Biological Sciences, U. Pittsburgh, Pittsburgh, PA 15260.
}

Many viruses employ proteolytic cleavage and conformational changes to transform the precursor procapsid into the mature virion [1]. Although cleavage often precedes the conformational changes. in some systems, the capsid matures without cleavage and, even when both processes occur, the relationship between them is poorly understood. Bacteriophage HK97 affords an opportunity to study these events. The conformational change, called expansion, involves subunit rotations that allow covalent crosslinks to form that stabilize the mature capsid (Head II) [2]. Cleavage involves removal of the $\mathrm{N}$-terminal $\Delta$-domain from the precursor capsid protein. A crystal structure exists for the mature capsid [3] and earlier states - uncleaved/unexpanded (Prohead I) and cleaved/unexpanded (Prohead II) - have been characterized by cryo-EM [4]. Nevertheless, basic questions persist: how does proteolysis trigger expansion? Is proteolysis necessary for expansion?

Whereas Prohead II may be induced to expand in vitro by acidic $\mathrm{pH}(\sim 4)$, Prohead I is unaffected by this treatment. However, we found that treating Prohead I with a more stringent perturbant, 5M urea, induces an expansion-like transition, as judged by mobility on native agarose gels. We analyzed the resulting particles - "Expanded Prohead I" - by cryo-EM and also determined Prohead I to same resolution. Focal pairs of micrographs were recorded on a FEI CM200-FEG operating at $120 \mathrm{keV}$; digitized, and reconstructed essentially as described [5]. The density maps have a resolution of $11 \AA$ (FSC; 0.3) and are compared in Figure 1 with Proheads I and II [2] at the same resolution.

These data show that, in Expanded Prohead I, expansion has not gone to completion. The facets of its icosahedral shell are not as flat as on Head II, but buckled; moreover the shell is thicker and has additional densities on its outer surface. The $\Delta$-domains are no longer visible beneath the capsomers, as on Prohead I. From these observations we infer that the $\Delta$-domains prevent premature expansion of Prohead I in addition to guiding the assembly of capsomers into Prohead I. Their removal by proteolysis, producing Prohead II, lowers the kinetic barrier to expansion. When expansion is initiated, the subunits rotate and the $\Delta$-domains, if still attached, become trapped at inter-capsomer interfaces. Thus a second function of $\Delta$-domain cleavage is that it enables expansion to proceed to completion, thereby allowing full cross-linking and optimal stabilization of the mature capsid.

\section{References}

[1] A. C. Steven et al., Curr Opin Struct Biol 15 (2005) 227.

[2] J. F. Conway et al., Science 292 (2001) 744.

[3] W. R. Wikoff et al., Science 289 (2000) 2129.

[4] J. F. Conway et al., J Mol Biol 253 (1995) 86.

[5] J. F. Conway and A. C. Steven, J Struct Biol 128 (1999) 106. 


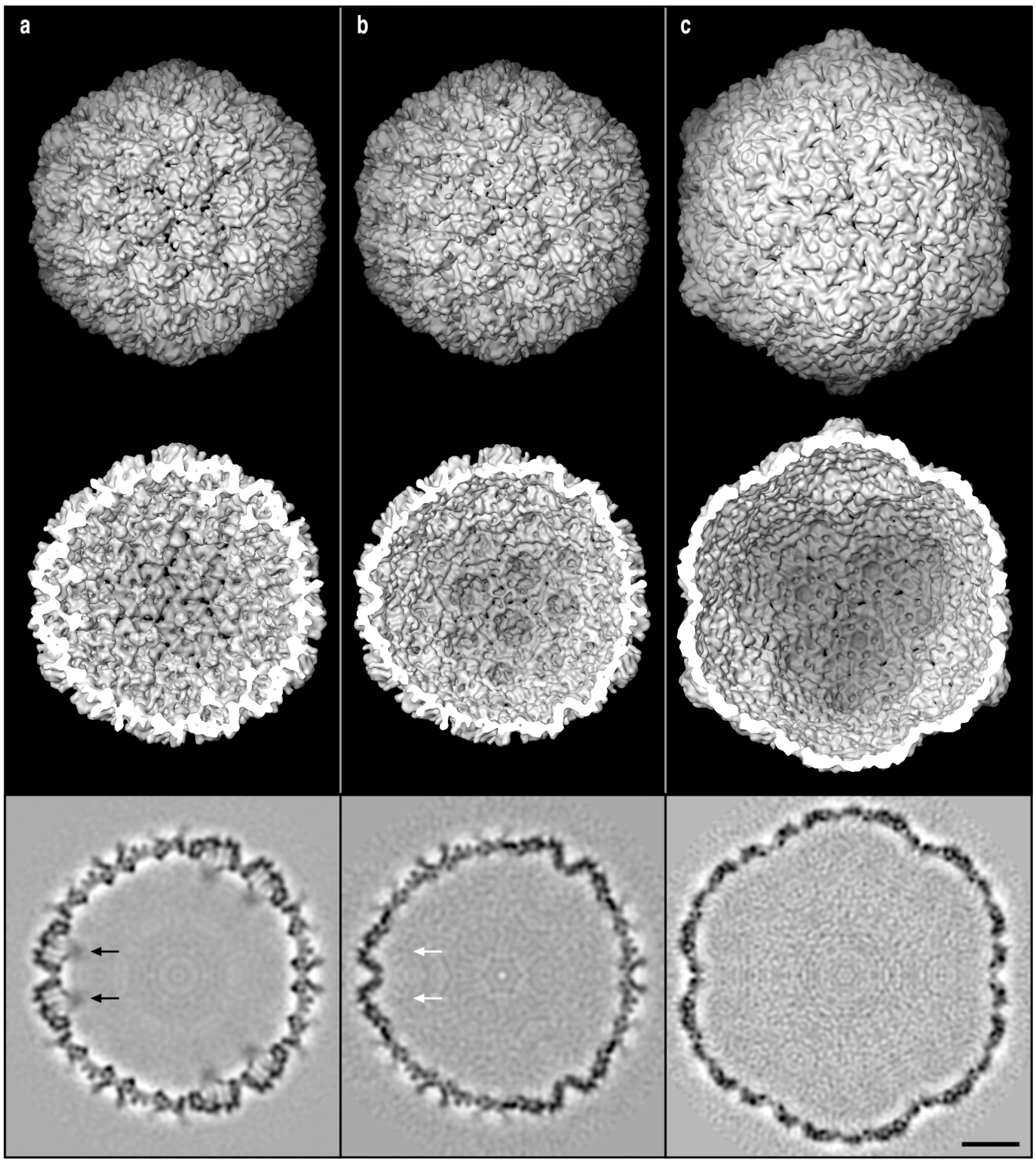

Figure 1. Surface views - exterior (top row) and interior (middle row) - and central sections (bottom row) of HK97 capsid reconstructions calculated from cryoEM data to a resolution of $11 \AA$, and viewed along an icosahedral 3-fold axis. (a) Prohead I, which has the 102-residue $\Delta$-domain at the $\mathrm{N}$-terminus of the capsid protein visible on the interior surface beneath the capsomer cavities (black arrows). (b) Prohead II, from which the $\Delta$-domains have been proteolytically removed. (c) Expanded

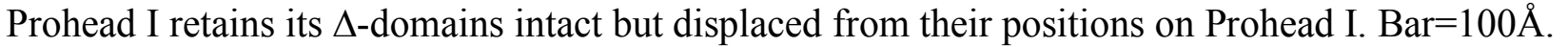

